Artículo

\title{
Incidencia de la participación ciudadana en educación en el contexto salvadoreño
}

\author{
Incidence of citizen participation in education in the Salvadoran context
}

\author{
Denisse Siliézar* \\ dni.szar@gmail.com \\ ISSN 1996-1642, Editorial Universidad Don Bosco, año 11, No.19, enero-junio de 2017, pp. 7-24 \\ Recibido: 22 de diciembre 2016. Aprobado: 22 de febrero de 2017
}

\begin{abstract}
Resumen
Dentro de la política social, y como parte de las medidas para lograr solventar problemas como la pobreza, desigualdad o exclusión, la educación desempeña un papel importante en la sociedad. Esto es así debido a que, entre otras cosas, permite un mayor conocimiento traducido en un mayor acceso a oportunidades de vida para las personas. Hablar de educación no implica solamente hablar del desarrollo económico de un país. Su importancia radica en la formación de ciudadanos responsables y conscientes sobre los problemas que atañen al resto de la sociedad y su significativa contribución en las soluciones. Este artículo propone que, si bien la educación por sí sola no puede a resolver los problemas sociales existentes, sí puede ser una buena herramienta de inicio por donde los cambios pueden comenzar a darse.
\end{abstract}

Palabras clave: educación, participación ciudadana, exclusión, currículo, pobreza.

\begin{abstract}
Either as part of a social policy or as a means to face problems like poverty, inequity or exclusion, education performs an important role in society. This is so due to, among other reasons; education allows a wider and deeper knowledge that becomes, in time, in better access to life opportunities for people. Education is important not only because it enhances the economical development of a country, but also because it shapes citizens that are responsible and mindful of their society problems and committed to solve such problems. In this paper, the author proposes that, even though education by itself cannot solve all social problems, it can be a powerful tool to boost changes in society.
\end{abstract}

Keywords: education, citizen participation, exclusion, curriculum, poverty.

* La autora es Licenciada en Economía. 


\section{Introducción}

Dentro de la política social, y como parte de las medidas para lograr afrontar problemas como la pobreza, la desigualdad o la exclusión, la educación desempeña un papel importante en la sociedad debido a que entre otras cosas, permite un mayor conocimiento traducido en un mayor acceso a oportunidades de vida para las personas. Hablar de educación no implica solamente hablar del desarrollo económico de un país. Su importancia radica en la formación de ciudadanos responsables y conscientes sobre los problemas que atañen al resto de la sociedad, y su significativa contribución a la misma.

El proceso de la educación se desarrolla desde diferentes aristas. Entre las más importantes se encuentran el currículo a desarrollar en los centros educativos, la metodología utilizada por los docentes dentro del salón de clases, el entorno familiar del estudiante, factores sociales externos como la inseguridad, la violencia y la pobreza, entre otros. A pesar de que estos elementos contribuyen desde su propia perspectiva al proceso educativo de los estudiantes, es necesario considerar como la educación puede mejorarse desde un conglomerado de estos factores, es decir, desde los procesos relacionados a la organización y la participación ciudadana.

En términos sencillos, la participación ciudadana hace referencia al trabajo en conjunto realizado por diferentes actores de la sociedad civil, como gobierno y organizaciones no gubernamentales que buscan alcanzar un mismo fin a partir de los distintos aportes o posturas propias. A nivel de país, el tema de participación es complejo debido a la falta de interés de la sociedad en general por este tipo de procesos. De acuerdo con el estudio "Cultura política de la democracia en El Salvador y en las Américas, 2014" del Proyecto de Opinión Pública de América Latina (LAPOP, por sus siglas en inglés), 3 de cada 10 salvadoreños suelen involucrarse en la resolución de problemas de su comunidad (Córdova, Macías, Cruz y Zechmeister, 2014), lo cual resulta preocupante ante la "indiferencia" de la población por la búsqueda de espacios que den cuenta de las necesidades más apremiantes de la sociedad a resolver.

Bajo esta noción, el tema de la participación ciudadana en educación adquiere relevancia debido a que implica el involucramiento de diferentes actores relacionados con la comunidad educativa, que buscan incidir en temas de la misma índole, ya sea participando a través de la formulación de propuestas, acciones y soluciones, capaces de solucionar problemas de interés común.

Debido a lo anterior, el propósito del presente trabajo es conocer de forma exploratoria la incidencia de la participación ciudadana en educación dentro del proceso educativo de los niños y jóvenes del país, con el fin de poder brindar propuestas y recomendaciones en pro de este tipo de mecanismos utilizados por la ciudadanía. Inicialmente, se realiza un pequeño recorrido teórico sobre el tema de participación, para luego describir con propiedad la participación ciudadana en educación, y culminar así en el tema a nivel de país; asimismo, se realiza un análisis sobre las valoraciones de padres y madres de familia

Incidencia de ciudadana en educación en el contexto

8. salvadoreño 
sobre la participación ciudadana en educación, a fin de obtener una breve aproximación sobre el funcionamiento de la misma a nivel de país, y su posible aplicación dentro de las actividades escolares.

\section{Marco conceptual Participación}

Inicialmente, la participación puede ser entendida como aquella acción necesaria para obtener algo, tratándose así de un derecho propio de cada persona (Rebollo y Martí, citado en Dueñas y García 2012). La participación como tal, comprende el movimiento constante de las personas, dejando de lado la idea de un "estado fijo" de las cosas al depender de factores como "el estado de ánimo, la disponibilidad del tiempo o el grado de compromiso que tienen las personas con su entorno" (Geilfus, citado en Dueñas y García 2012). De esta forma, se podrá identificar una escala de niveles de participación de la persona a saber: 1. Pasividad (Nivel más sencillo); 2. Suministro de información; 3. Participación por consulta; 4. Participación por incentivos; 5. Participación funcional; 6. Participación interactiva; y 7. Autodesarrollo (Nivel más complejo).

La participación implica ser partícipe de algo, en donde es posible expresar opiniones y propuestas a fin de lograr un acuerdo en conjunto sobre determinada situación. De acuerdo con Cunill (citado en Villareal, 2010), desde el ámbito público la participación puede presentar la siguiente tipología:

Participación Social: Es la que se da en razón de la pertenencia del individuo a asociaciones u organizaciones para la defensa de los intereses de sus integrantes, y el interlocutor principal. No es el Estado, sino otras instituciones sociales.

Participación Comunitaria: Es el involucramiento de individuos en la acción colectiva que tiene como fin el desarrollo de la comunidad mediante la atención de las necesidades de sus miembros y asegurar la reproducción social; suele identificarse con la beneficencia. El interlocutor principal de estas acciones no es el Estado y, en todo caso, lo que se espera de él es recibir apoyo asistencial.

Participación Política: Tiene que ver con el involucramiento de los ciudadanos en las organizaciones formales y mecanismos del sistema político: partidos, parlamentos, ayuntamientos, elecciones. Es una participación mediada por los mecanismos de la representación política.

Participación Ciudadana: Es aquélla en la que los ciudadanos se involucran de manera directa en acciones públicas con una concepción amplia de lo políico y una visión del espacio público como espacio de ciudadanos. Esta participación pone en contacto a los ciudadanos y al Estado en la definición de las metas colectivas y las formas de alcanzarlas.

Estos procesos requieren un rol activo por parte de la ciudadanía a fin de poder "garantizar el bien común y la cohesión de la sociedad en torno a sus valores"

9. Diálogos 
(Tamayo, 2013 p. 19). Esto también implica relaciones de poder en tanto que convergen dentro de la sociedad diferentes aportes $u$ opiniones de diversos actores de la sociedad.

\section{Participación ciudadana}

De manera práctica, la participación ciudadana puede entenderse como el involucramiento de la sociedad civil en distintos problemas de índole pública y política, donde podrán definirse propuestas y acciones necesarias para dar solución a determinado problema identificado.

De acuerdo con Merino (citado en Dueñas y García 2012), la participación ciudadana significa intervenir en los centros de gobiernos de una colectividad, participar en sus decisiones en la vida colectiva, de la administración de sus recursos, del modo como se distribuye sus costos y beneficios. El propósito es lograr que la población influya sobre las políticas y decisiones públicas, siendo necesario institucionalizar mecanismos, procesos y organismos a través de una normatividad legal.

La participación ciudadana al igual que otro tipo de participación, conlleva un nivel en la participación ejercida por las personas dentro de la sociedad. Al respecto, Hambleton y Hogget (citado en Guillén, Sáenz, Badii, y Castillo, 2009) desarrollaron una actualización de la escalera de participación que demuestra las formas de participación ciudadana divididos en tres áreas correspondientes.

Tabla 1. Formas y niveles de participación ciudadana según Hambleton y Hogget (1994).

\begin{tabular}{cll}
\hline Nivel & Forma de Participación & Nivel de Participación \\
\hline 12 & Control Independiente & Control Ciudadano \\
11 & Control basado en la Confianza & \\
\hline 10 & Control Delegado & \\
09 & Co-participación & \\
08 & Descentralización limitada & Participación Ciudadana \\
07 & Consejos de Accesoria Efectiva & \\
06 & Consulta Genuina & \\
05 & Información de Alta Calidad & \\
\hline 04 & Cuidado Ciudadanía & No Participación Ciudadana \\
03 & Información Pobre & \\
02 & Consulta Cínica & \\
01 & Decepción Ciudadana & \\
\hline
\end{tabular}

Incidencia de la participación ciudadana en educación en el contexto

10. salvadoreño
De acuerdo con ambos autores, las formas de participación del nivel 1 al 4 corresponden a un nivel nulo de participación ciudadana dada la poca información existente; generando así poco interés en la población a participar ante el bombardeo de campañas publicitarias. En el siguiente nivel, la existencia 
de información de calidad permite un mayor involucramiento de la sociedad, debido a que permite escenarios de "discusión y negociación sobre la actuación gubernamental". Finalmente, el nivel de control ciudadano implica la participación entera de la sociedad civil, llegando a tener "control y poder de decisión" sin coaccionar las acciones o planteamientos de otros actores protagonistas.

El ejercicio de la participación ciudadana permite, entonces, un mayor conocimiento sobre determinada situación dado que se retoman distintos puntos de vista provenientes de diferentes protagonistas así como también, transparencia en cuanto a los procesos formulados y decisiones tomadas provenientes de las autoridades de gobierno, representantes de la sociedad civil, entre otros. La participación ciudadana se vuelve así, "un requisito para el ejercicio de la democracia y para la incidencia activa y protagónica de la sociedad en la toma de decisiones" (Varela, 2014).

Finalmente, con miras a que este tipo de participación pueda darse, es importante que existan condiciones necesarias para su adecuado funcionamiento, es decir, es importante contar con mecanismos que permitan que la opinión ciudadana llegue a entidades de alto nivel para que la información pueda ser discutida entre ambas partes. A manera de ejemplo, y desde una perspectiva de la gestión pública municipal, se pueden mencionar los siguientes mecanismos de participación ciudadana: las Sesiones Públicas del Concejo Municipal, los Comités de Desarrollo Local (CDL), el Presupuesto de Inversión Participativa, la Consulta Popular, el Cabildo Abierto, el Plan de Inversión Participativo y la Consulta Vecinal Sectorial (FUNDE, 2008).

\section{Participación ciudadana en educación}

De acuerdo con Torres (2001), la participación ciudadana en educación desde una perspectiva amplia debe comenzar aceptando los siguientes elementos:

La educación: no se limita a la educación escolar, ni los aprendizajes necesarios -para la vida, para el trabajo, para la participación, para la ciudadanía plenapueden limitarse a un período determinado de la vida de una persona. El aprendizaje se inicia con el nacimiento y se extiende a lo largo de toda la vida. Arranca en el hogar, antecede y excede a la institución escolar, abarcando un amplio conjunto de instituciones, modalidades, relaciones y prácticas.

La sociedad civil: es una realidad sumamente heterogénea y compleja, formada por un amplio mosaico de organizaciones (siendo las ONGs apenas un segmento, y minoritario, de las OSC) en la que se expresan múltiples visiones, intereses y conflictos.

La participación: para convertirse en instrumento de desarrollo, empoderamiento y equidad social debe ser significativa y auténtica; involucrar a todos los actores, diferenciando pero sincronizando sus roles; y darse en los diversos ámbitos y dimensiones de lo educativo: desde el aula de clase hasta la política educativa, 
dentro de la educación escolar y también de la extra-escolar, en los aspectos administrativos y también en los relacionados con la enseñanza y el aprendizaje, a nivel local así como a nivel nacional y global.

Partiendo de los elementos anteriores, la participación ciudadana en educación implica la organización e instrumentación de los servicios que se ofrecen en las escuelas, donde diferentes actores se involucran en la planeación, el seguimiento y valoración de las acciones educativas a fin de optimizar la calidad de servicio prestado (Secretaría de Educación Pública de México, 2010).

Por otra parte, dado que el tema de educación es un tema que no solo corresponde a un área específica, es necesario considerar algunos elementos comúnmente presentes en el proceso educativo. Al respecto, Torres (2001) señala que el proceso educativo al ser "un derecho, un bien público y asunto de todos" (p.16), conlleva tomar en cuenta tanto actores, espacios, niveles, dimensiones, ámbitos y alcances.

Tabla 2: Elementos relacionados a la participación ciudadana en educación según Torres (2001).

\begin{tabular}{|c|c|c|c|c|c|}
\hline Actores & Espacios & Niveles & Dimensiones & Ámbitos & Alcances \\
\hline $\begin{array}{l}\text { Alumnos } \\
\text { Padres de } \\
\text { familia } \\
\text { Docentes } \\
\cdot \text { comunitarias } \\
\text { - identitarias } \\
\text { - académicas } \\
\text { - filantrópicas } \\
\text { Sindicatos } \\
\text { Mov. Sociales } \\
\text { Part. políticos } \\
\text { Voluntariado } \\
\text { Medios Redes } \\
\text { Otros }\end{array}$ & $\begin{array}{l}\text { Aula } \\
\text { Institución } \\
\text { escolar } \\
\text { Red escolar } \\
\text { Sistema } \\
\text { escolar } \\
\text { Sistema } \\
\text { educativo } \\
\text { Política } \\
\text { educativa }\end{array}$ & $\begin{array}{l}\text { Acceso/ } \\
\text { Asistencia/Uso } \\
\text { Información } \\
\text { Comunicación } \\
\text { Consulta } \\
\text { Decisión }\end{array}$ & $\begin{array}{l}\text { Administrativo } \\
\text { Curricular } \\
\text { Pedagógico }\end{array}$ & $\begin{array}{l}\text { Diagnóstico } \\
\text { y prospectiva } \\
\text { Planificación } \\
\text { Diseño } \\
\text { Ejecución } \\
\text { Seguimiento } \\
\text { Sistematización } \\
\text { Evaluación } \\
\text { Interpretación, } \\
\text { control y uso } \\
\text { de resultados }\end{array}$ & $\begin{array}{l}\text { Sub-nacional } \\
\text { (estadual, } \\
\text { municipal, } \\
\text { local) } \\
\text { Nacional } \\
\text { Supra- } \\
\text { nacional } \\
\text { (regional, } \\
\text { continental, } \\
\text { mundial) }\end{array}$ \\
\hline
\end{tabular}

De igual forma, es importante reconocer que existen diferentes grados de aporte dentro del ámbito de la participación educativa. Al respecto, el "Estudio cualitativo sobre la participación ciudadana en el mejoramiento de la calidad de la educación en cinco países latinoamericanos", elaborado por el Compromiso Cívico para la Reforma de la Educación en Centroamérica (CERCA, 2004), provee una escala de los niveles de participación detalladas en la tabla siguiente. 
Tabla 3: Escala de niveles de participación según CERCA (2004).

\begin{tabular}{l|l}
\hline ÓPTIMO & $\begin{array}{l}\text { Nivel alto más: *Exige rendimiento / rendición de cuentas en sentido amplio. } \\
\text { *Participa organizadamente en la gestión pedagógica y procesos de aprendizaje. } \\
\text { *Organización con redes locales y regionales para lograr cambios de políticas. } \\
\text { *Participa efectivamente en el mejoramiento de la calidad de la educación. }\end{array}$ \\
\hline ALTO & $\begin{array}{l}\text { Nivel medio más: *Monitorea el rendimiento y rendición de cuentas a nivel } \\
\text { de la escuela. *Participa en la gestión administrativa. *Apoya en las tareas de } \\
\text { sus hijos. *Apoya en la elaboración de materiales didácticos. *Sabe cómo } \\
\text { apoyar en el mejoramiento de la calidad de la educación. }\end{array}$ \\
\hline MEDIO & $\begin{array}{l}\text { Nivel básico más: *Participa organizadamente en la escuela. *Solicita } \\
\text { información. *Esfuerzos colectivos para el mejoramiento de la infraestructura } \\
\text { y servicios. *Consciente de la importancia de la educación y de su rol. }\end{array}$ \\
\hline BÁSICO & $\begin{array}{l}\text { Participación Individual a solicitud de directivos escolares. *Aporta y } \\
\text { gestiona recursos materiales solicitados. *Responde a invitación de } \\
\text { docentes. *Recibe información. *Sensible a las necesidades educativas }\end{array}$ \\
\hline NULO & $\begin{array}{l}\text { *Manda a su hijo a la escuela. *No se relaciona con actividades } \\
\text { de la escuela. }\end{array}$ \\
\hline
\end{tabular}

De acuerdo con los datos de la tabla anterior, cada nivel de participación toma en cuenta elementos asociados a la "iniciativa propia, conciencia de derechos ciudadanos, información y grado de incidencia" importantes en el desarrollo y calidad educativa del estudiante.

Por otra parte, los mecanismos de participación utilizados en educación serán diferentes según el contexto del que se hable, los tipos de actores involucrados, conocimientos adquiridos. A manera de ejemplo, la municipalidad de Providencia en Chile detalla cuatro mecanismos de participación utilizados por el municipio que ilustran el funcionamiento de este tipo de procesos.

Tabla 4: Mecanismos de participación ciudadana en educación según la Corporación de Desarrollo Social de Providencia (2016)

\begin{tabular}{|c|c|c|}
\hline Mecanismo & Descripción & Requisitos para participar \\
\hline 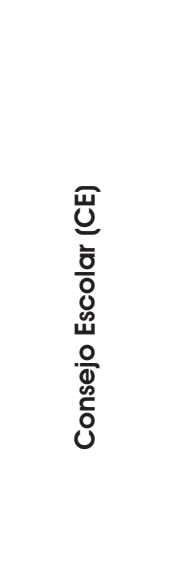 & $\begin{array}{l}\text { El principal propósito que tiene el CE es producir } \\
\text { un espacio que articule y exprese, reflexión y } \\
\text { diálogo entre los miembros que constituyen } \\
\text { la comunidad escolar en relación a temas } \\
\text { pertinentes y relevantes en el ámbito escolar en } \\
\text { el establecimiento educacional. El CE tendrá } \\
\text { carácter informativo, consultivo y propositivo, salvo } \\
\text { que el sostenedor decida darle carácter resolutivo. } \\
\text { El CE será informado a lo menos de las siguientes } \\
\text { materias: a) Los logros de aprendizaje de los } \\
\text { alumnos. b) Informes de las visitas inspectivas del } \\
\text { Ministerio de Educación, entre otros. El CE será } \\
\text { consultado a lo menos en los siguientes } \\
\text { aspectos: a) Proyecto Educativo Institucional. } \\
\text { b) Programación Anual y actividades } \\
\text { extracurriculares. c) Las metas del establecimiento } \\
\text { y los proyectos de mejoramiento propuestos; }\end{array}$ & $\begin{array}{l}\text { En cada establecimiento } \\
\text { educacional subvencionado } \\
\text { deberá existir un CE que } \\
\text { será un órgano integrado } \\
\text { a los menos por el director } \\
\text { del establecimiento que lo } \\
\text { presidirá; por el sostenedor o } \\
\text { un representante designado } \\
\text { por él; un docente } \\
\text { elegido por los profesores } \\
\text { del establecimiento; el } \\
\text { presidente del centro de } \\
\text { padres y apoderados, y el } \\
\text { presidente del centro de } \\
\text { alumnos en el caso que el } \\
\text { establecimiento imparta } \\
\text { enseñanza media. }\end{array}$ \\
\hline
\end{tabular}




\begin{tabular}{|c|c|c|}
\hline 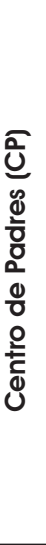 & $\begin{array}{l}\text { Los CP y Apoderados, son organismos que } \\
\text { comparten y colaboran en los propósitos } \\
\text { educativos y sociales de los establecimientos } \\
\text { educacionales de que forma parte. Para esto se } \\
\text { plantean los siguientes objetivos: a) Fomentar la } \\
\text { preocupación de sus miembros por la formación } \\
\text { y desarrollo personal de sus hijos y pupilos y, en } \\
\text { consonancia con ello, promover las acciones de } \\
\text { estudio y capacitación que sean convenientes } \\
\text { para el mejor cometido de las responsabilidades } \\
\text { educativas de la familia. b) Integrar activamente } \\
\text { a sus miembros en una comunidad inspirada por } \\
\text { principios, valores e ideales educativos comunes, } \\
\text { canalizando para ellos las aptitudes, intereses } \\
\text { y capacidades personales de cada uno; entre } \\
\text { otros. }\end{array}$ & $\begin{array}{l}\text { Pertenecerán al CP de cada } \\
\text { establecimiento educacional } \\
\text { los padres y apoderados } \\
\text { del mismo. También podrán } \\
\text { participar en calidad de } \\
\text { cooperadores, las personas } \\
\text { naturales o jurídicas que se } \\
\text { comprometan a contribuir al } \\
\text { cumplimiento de los fines del } \\
\text { Centro de Padres. }\end{array}$ \\
\hline 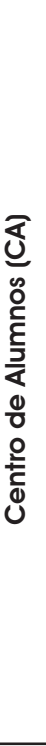 & $\begin{array}{l}\text { El CA es la organización formada por los estudi- } \\
\text { antes de segundo ciclo de enseñanza básica } \\
\text { y enseñanza media, de cada establecimiento } \\
\text { educacional. Su finalidad es servir a sus miembros } \\
\text { como medio para desarrollar en ellos el pensa- } \\
\text { miento reflexivo, el juicio crítico y la voluntad de } \\
\text { acción; de formarlos para la vida democrática, } \\
\text { y de prepararlos para participar en los cambios } \\
\text { culturales y sociales. En ningún establecimiento } \\
\text { se podrá negar la constitución y funcionamiento } \\
\text { de un CA. Las funciones del CA son las siguien- } \\
\text { tes: a) Promover la creación e incremento de } \\
\text { oportunidades para que los alumnos manifiesten } \\
\text { democrática y organizadamente sus intereses, } \\
\text { inquietudes y aspiraciones. b) Promover en el } \\
\text { alumnado la mayor dedicación a su trabajo } \\
\text { escolar, procurando que se desarrolle y fortalezca } \\
\text { un adecuado ambiente educativo y una estrecha } \\
\text { relación humana entre sus integrantes basada en } \\
\text { el respeto mutuo; entre otros. }\end{array}$ & $\begin{array}{l}\text { El requisito básico para } \\
\text { participar en un CA es ser } \\
\text { alumno regular del estab- } \\
\text { lecimiento al que pertenece } \\
\text { el CA. La ley especifica que } \\
\text { cada CA se estructurará a } \\
\text { lo menos, con los siguientes } \\
\text { organismos: a) La Asamblea } \\
\text { General b) La Directiva c) El } \\
\text { Consejo de Delegados de } \\
\text { Curso d) El Consejo de Curso } \\
\text { e) La Junta Electoral. Tam- } \\
\text { bién formarán parte consti- } \\
\text { tutiva del CA todos aquellos } \\
\text { organismos y comisiones de } \\
\text { tipo funcional, permanentes } \\
\text { o circunstanciales, que para } \\
\text { el mejor cumplimiento de } \\
\text { sus funciones puedan crear } \\
\text { la Asamblea General, la } \\
\text { Directiva del CA o el Consejo } \\
\text { de Delegados de Curso. }\end{array}$ \\
\hline 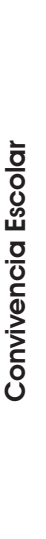 & $\begin{array}{l}\text { Orienta las acciones, iniciativas y programas que } \\
\text { promuevan y fomenten la comprensión y el desar- } \\
\text { rollo de una convivencia inclusiva, participativa, } \\
\text { solidaria, tolerante, pacífica y respetuosa, en un } \\
\text { marco de equidad de género y con enfoque de } \\
\text { derechos. 1).- Fortalecer la dimensión formativa } \\
\text { de la Convivencia Escolar como componente } \\
\text { central en la organización disciplinaria y de la } \\
\text { gestión institucional. 2).- Promover el compromiso } \\
\text { y la participación de la Comunidad Educativa, en } \\
\text { la construcción de un proyecto institucional que } \\
\text { tenga como componente central la Convivencia } \\
\text { Escolar, y el ejercicio de los derechos y deberes } \\
\text { de cada uno de los actores; entre otros. }\end{array}$ & $\begin{array}{l}\text { Ser parte de la Comunidad } \\
\text { Educativa, específicamente: } \\
\text { los alumnos, alumnas, pa- } \\
\text { dres, madres y apoderados, } \\
\text { profesionales y asistentes de } \\
\text { la educación, así como los } \\
\text { equipos docentes y directivos } \\
\text { de los establecimientos edu- } \\
\text { cacionales, La participación } \\
\text { de la Comunidad Educativa } \\
\text { está garantizada por ley, es- } \\
\text { pecialmente a través de los } \\
\text { centros de alumnos, centros } \\
\text { de padres y apoderados, } \\
\text { consejos de profesores y } \\
\text { consejos escolares }\end{array}$ \\
\hline
\end{tabular}

Incidencia de la participación ciudadana en educación en el contexto

14. salvadoreño

\section{Participación ciudadana en educación en El Salvador}

Como se describió anteriormente, los procesos de participación toman en cuenta diferentes elementos relacionados a un mismo fin. En cuanto al ámbito educativo se refiere, el "Informe de Participación Ciudadana en la Gestión Pública 2013" (2014) de la Secretaría de Asuntos Estratégicos de la Presidencia, señala 
que el Ministerio de Educación contiene las siguientes iniciativas en materia de gestión participativa de los ciudadanos.

Consejos consultivos Son espacios de participación ciudadana para la implementación y retroalimentación de las políticas educativas, sus programas y proyectos. Constituidos por madres, padres de familia, docentes, directores, estudiantado, iglesias, gremios, ONG, entre otros; estos se eligen democráticamente y constituyen el enlace entre las comunidades educativas y el Ministerio de Educación. Los consejos consultan, delibera, y presentan propuestas desde la realidad del territorio y a su vez comparten la información sobre la gestión escolar con las comunidades del entorno. Son estructuras flexibles y adaptables a la realidad organizativa de los territorios.

Escuela inclusiva de tiempo pleno Desde la implementación del plan social educativo "Vamos a Escuela" se concibe una administración escolar con enfoque participativo, bajo el planteamiento de escuela inclusiva de Tiempo Pleno, un modelo educativo que cuenta con tres componentes: uno pedagógico, uno territorial y uno organizativo. Este último contempla la participación de la comunidad como un eje fundamental del proceso de enseñanza-aprendizaje.

Rendición de cuentas en centros escolares Es el mecanismo a través del cual los organismos de administración escolar informan a la comunidad educativa los resultados obtenidos y el uso de los recursos asignados a los centros escolares para el año lectivo. La rendición de cuentas no solo permite informar y evaluar los logros y dificultades, sino también es un espacio de interacción con la comunidad del entorno, entablando un dialogo con estudiantes, docentes, padres, madres, lideres, líderes comunitarios, autoridades locales, cooperantes, iglesias y consejos consultivos, para que estos puedan evaluar y proponer acciones de mejora.

En cuanto al tema de transparencia se refiere, la Corte de Cuentas de la República y el Ministerio de Educación desarrollan en conjunto el plan piloto "Programa de Contraloría Escolar", el cual busca la inclusión de jóvenes estudiantes en el "control social, como parte de las acciones que la entidad fiscalizadora del Estado realiza" (Corte de Cuentas, 2016). El programa, pretende generar conciencia en cuanto a la necesidad de "ejercer control y fiscalización de los bienes y recursos destinados a centros escolares, siendo estos bienes públicos" Corte de Cuentas, 2016). De acuerdo con la información provista por la Dirección de Transparencia (Ministerio de Educación, 2016), el programa ha sido implementado en ocho centros educativos a nivel nacional, habiéndose realizado para Julio 2016 elecciones para nombrar los contralores escolares de cada centro educativo.

En este sentido, el papel del estudiantado es retomado dentro de los procesos participativos relacionados al ámbito educativo. Para el Ministerio de Educación (2016), la participación estudiantil será "la forma en que el sector estudiantil ejerce la gobernabilidad y contribuye al logro de los objetivos planteados en el 
Proyecto Estratégico de la Escuela". El propósito de este tipo de participación, es "que las y los estudiantes se sensibilicen, reconozcan y practiquen procesos democráticos de organización social en pro de sus derechos y cumplimiento de sus deberes para la mejora de los ambientes de convivencia escolar y optimizar el buen uso de los bienes públicos" (Ministerio de Educación, 2016).

Entre las principales funciones a realizar por los estudiantes estarán las siguientes:

1. Elaborar el plan de trabajo, apoyando las áreas de convivencia escolar, descubriendo valores.

2. Dar seguimiento y evaluar el desarrollo del plan de trabajo. Informando constantemente a los miembros de la comunidad educativa.

3. Promover el cumplimiento de los deberes y derechos del sector, especialmente, para el logro de la efectividad del centro educativo.

4. Informar al sector estudiantil lo acordado en el Consejo Directivo Escolar.

5. Asesorar, orientar y apoyar el trabajo de otras organizaciones estudiantiles existentes en el centro escolar y conformar los comités siguientes: a) Comité de convivencia que debe garantizar el funcionamiento del manual de convivencia. b) Protocolo de bienvenida. c) Club de tutoría académico. d) Comité de mediación escolar. e) Comité de gestión de medio ambiente y prevención de desastres. f) Implementar los itinerarios pedagógicos para descubrir valores, programa de educación familiar. g) Apoyar a cada sector de la comunidad educativa para realizar actividades que ayuden a mejorar el centro escolar y convivencia. h) Participar en las acciones de evaluación de la participación estudiantil.

Otros mecanismos también presentes dentro del proceso educativo son los siguientes (Ministerio de Educación, 2016):

Feria de paquetes escolares

- Objetivo: Facilitar a los integrantes de los organismos de administración escolar de los Consejos Directivos Escolares (CDE), los Consejos Educativos Católicos Escolares (CECE) y los Consejos Institucionales Educativos (CIE) beneficiados con el programa de paquete escolar, los procesos de selección y contratación de los bienes y servicios (confección de uniformes, zapatos y paquete de utiles escolares), para disminuir el tiempo invertido en los procesos de compra y dedicar más tiempo a las actividades pedagógicas en los centros escolares.

- Integrantes: Ministerio de Educación, Organismos de Administracion Escolar, Padres de Familia, Estudiantes de centros educativos, Personas proveedoras de útiles, zapatos y servicios de confeccion de uniformes, entre otros.

Programa de Alimentación y Salud Escolar (PASE)

Incidencia de la participación ciudadana en educación en el contexto

16. salvadoreño
- Requisitos: Su implementación en cada centro educativo requiere de una participación activa y decidida de la comunidad educativa, quien debe de formar parte de su ejecución y toma de decisiones. El organismo de administración escolar, quien es la máxima autoridad dentro del centro 
educativo, debe a través de las asambleas de padres y madres de familia, convocar a la conformación y organización de comités de desarrollo educativo, en este caso para apoyo al pase, definiéndole funciones a través de las comisiones en apoyo a la actividades del programa, para favorecer su ejecución.

- Papel de la participación: La participación ciudadana se promueve en el centro educativo, para resolver todas las necesidades que se presentan en la ejecución del programa, que va desde retirar los alimentos en los centros de distribución según las convocatorias, la preparación de los mismos, manejos de la bodega, controles de inventario, lavado de platos, aportes de productos mismos, manejo de la bodega, controles de inventario, lavado de platos, aportes de productos complementarios, huertos escolares, o cualquier otra necesidad que se presente para garantizar una buena ejecución.

- Transparencia: Cuando se logra una buena participacion aparte de lograr una mejor ejecución del programa, tambien se contribuye a transparentar lo que sucede en la escuela con respecto a la asignación de los alimentos, pues la comunidad educativa en su totalidad ejerce su rol de contraloría social al estar presente en todas las acciones del programa.

De acuerdo con la información presentada anteriormente, los mecanismos que acompañan a la participación ciudadana en educación son diversos y complejos en alguna forma, por lo que es importante no olvidar los verdaderos actores de mayor protagonismo en el tema; es decir, los alumnos de los centros escolares. Incentivar este tipo de prácticas participativas no solamente permite alcanzar algún tipo de consenso, sino que también fortalece los procesos de integración social e impulsa el desarrollo de cada persona como individuo capaz de ejercer y tomar decisiones por cuenta propia. Este tipo de herramientas participativas permiten el empoderamiento de la sociedad, favoreciendo en última instancia la inclusión social de los habitantes del país en vísperas de acceder a una mejor calidad de vida.

\section{Método de estudio}

Desde un enfoque metodológico cuantitativo desarrollado con estadística descriptiva y el uso de cuestionarios, en la presente investigación se aplicaron 19 encuestas en línea a Padres de Familia cuyos hijos atienden Instituciones educativas de tipo público y privado, en los niveles escolares que comprenden desde primer grado hasta bachillerato con el fin de diversificar la información y tener una mayor amplitud del tema. Dentro de la encuesta, se recopiló inicialmente información de tipo general para después realizar preguntas de interés sobre su conocimiento del tema. El tipo de preguntas utilizadas fueron de tipos abiertas, cerradas, opción múltiple, y de escala de likert. 


\section{Análisis de los datos y resultados}

De acuerdo con los datos recopilados, el $52.6 \%$ corresponde al género femenino y $47.4 \%$ al género masculino. En cuanto al estado civil se refiere, el $63.2 \%$ se encuentra casado/a, el $21.1 \%$ soltero/a, 10.5\% acompañado/a, y 5.3\% divorciado/a. Por otra parte, en cuanto al tipo de institución educativa atendida por sus hijos, los datos señalan una mayor predominancia del sector privado (89.6\%), a diferencia del sector público (10.5\%). El nivel de estudios con mayor representatividad, es el que comprende de primer grado a tercer grado con un valor de $47.4 \%$, seguido por séptimo grado a noveno grado con un $21.1 \%$, cuarto grado a sexto grado con un $15.8 \%$, y bachillerato también con un $15.8 \%$.

Gráfico 1. Género y estado civil de los encuestados

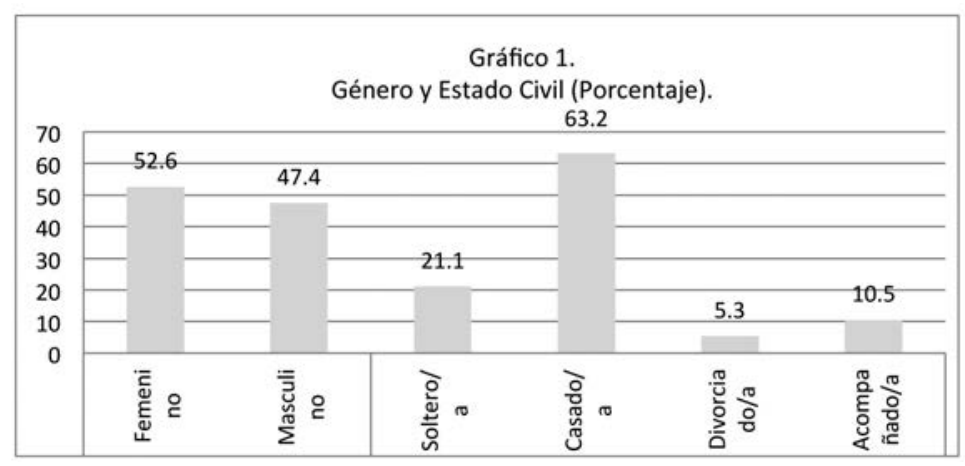

Gráfico 2. Tipo de institución educativa y grado de estudio de los hijos

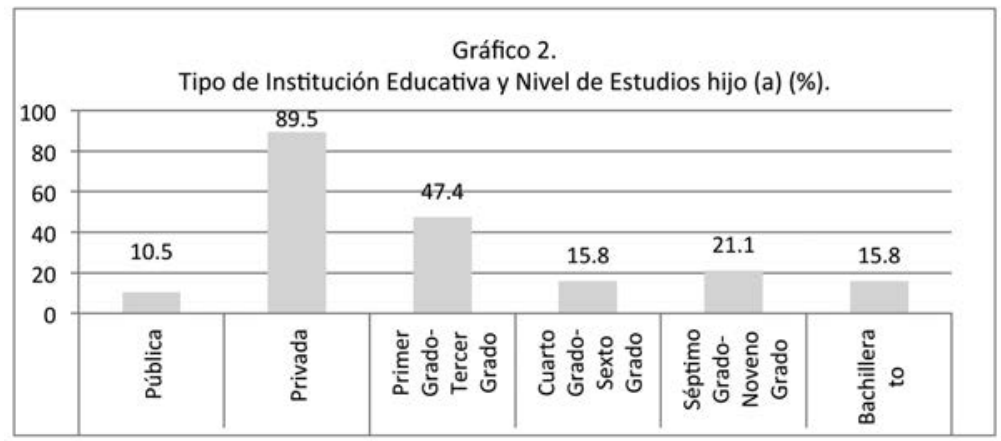

Inicialmente, dado que el tema de participación ciudadana en educación implica el involucramiento de diferentes sectores en vistas a mejorar la calidad educativa de los estudiantes, es necesario considerar la valoración de los padres de familia en cuanto a si participan o no en la educación de sus hijos. Ante esto, la pregunta inicial realizada fue la siguiente: ¿Participa usted activamente en cualquier actividad relacionada con la educación de su hijo(a)? 
Tabla 5. Participación del padre de familia en actividades educativas de los hijos.

\begin{tabular}{cc}
\hline $\begin{array}{c}\text { ¿Participa usted activamente en cualquier actividad } \\
\text { relacionada con la educación de su hijo(a)? }\end{array}$ \\
\hline Sí & $89,5 \%$ \\
No & $10,5 \%$ \\
\hline Total & $100,0 \%$ \\
\hline
\end{tabular}

De acuerdo con los datos, el $89.5 \%$ de los padres de familia entrevistados manifestaban participar activamente en actividades relacionadas a la educación de sus hijos, mientras que un 10.5\% manifestó no hacerlo. Las personas participantes mencionaban la importancia de involucrarse en las actividades de sus hijos, dado que es una fuente de apoyo a su desarrollo, motivación para su vida, así como formación de su educación integral. Las personas que aducían no participar, explicaban que por razones familiares y compromisos de trabajo, se hacía difícil involucrarse en este tipo de actividades relacionadas a la educación de sus hijos.

Por otra parte, en lo que respecta a los mecanismos de participación ciudadana relacionados a la educación, los padres de familia manifestaron conocer los siguientes:

Tabla 6. Tipos de participación ciudadana

\begin{tabular}{lc}
\hline \multicolumn{2}{c}{$\begin{array}{c}\text { De las siguientes opciones, señale los tipos de } \\
\text { participación ciudadana que usted conoce: }\end{array}$} \\
\hline Consejos escolares & $57.9 \%$ \\
Consejos de Padres y madres de Familia & $94.7 \%$ \\
Consejos de alumnos & $57.9 \%$ \\
\hline Otros & $5.3 \%$ \\
\hline
\end{tabular}

Como se puede observar en la tabla anterior, los consejos de padres y madres de familia son el mecanismo que mayormente se conoce, es decir, $94.7 \%$ de las personas entrevistadas manifestaron saber de la existencia de este mecanismo. De igual forma, los consejos escolares y de alumnos resultan conocidos para la población, con un valor de $57.9 \%$ en cada caso. En cuanto a la variable de otros y del total de encuestas realizadas, un padre de familia mencionó la existencia de un comité de ayuda comunitaria.

Debido a que la participación de los padres de familia en la educación de sus hijos, puede ser realizada bajo distintas formas o niveles, fue utilizada dentro de la encuesta la escala de niveles provista por el "Estudio cualitativo sobre la participación ciudadana en el mejoramiento de la calidad de la educación en cinco países latinoamericanos", elaborado por el Compromiso Cívico para la Reforma de la Educación en Centroamérica (CERCA, 2004), con el fin de 
determinar y dar una valoración más acertada sobre el tipo de participación realizada.

Debe mencionarse, que los niveles de participación fueron adecuados dentro de la encuesta para una mejor comprensión por parte de las personas encuestadas. Los niveles de participación utilizados dentro de la encuesta fueron cuatro (óptimo, alto, medio, y básico), y para conocer mayor detalle de cada uno, estos fueron evaluados bajo una escala de tipo likert cuyos resultados se detallan en la tabla siguiente.

Tabla 7. Nivel de participación de padres y madres en la educación de los hijos.

\begin{tabular}{|c|c|c|c|}
\hline \multicolumn{4}{|c|}{$\begin{array}{c}\text { ¿Cómo considera que es su nivel de participación en la educación } \\
\text { de su hijo(a) según la siguiente clasificación? }\end{array}$} \\
\hline \multirow[t]{4}{*}{ ÓPTIMO } & \multirow{4}{*}{$\begin{array}{l}\text { 1. Está al pendiente del rendimiento escolar } \\
\text { provisto por la institución educativa a su hijo } \\
\text { (a), así como de los procesos que involucran } \\
\text { la rendición de cuentas; Se organiza de } \\
\text { forma local entre la comunidad para } \\
\text { lograr cambios o sugerencias de políticas, } \\
\text { en pro del beneficio de su hijo (a). }\end{array}$} & Indiferente & $15.8 \%$ \\
\hline & & De acuerdo & $57.9 \%$ \\
\hline & & Totalmente de acuerdo & $26.3 \%$ \\
\hline & & Total & $100.0 \%$ \\
\hline \multirow[t]{4}{*}{ ALTO } & \multirow{4}{*}{$\begin{array}{l}\text { 2. Apoya la realización de tareas de sus } \\
\text { hijos; Apoya en la elaboración de materiales } \\
\text { didácticos que se puedan necesitar en } \\
\text { la institución educativa, y que mejoren la } \\
\text { calidad de la educación de su hijo (a). }\end{array}$} & Indiferente & $10.5 \%$ \\
\hline & & De acuerdo & $42.1 \%$ \\
\hline & & Totalmente de acuerdo & $47.4 \%$ \\
\hline & & Total & $100 \%$ \\
\hline \multirow[t]{6}{*}{ MEDIO } & \multirow{6}{*}{$\begin{array}{l}\text { 3. Participa organizadamente dentro } \\
\text { de las tareas que requiere la institución } \\
\text { educativa, ya sea solicitando información, } \\
\text { o realizando trabajo en conjunto con los } \\
\text { demás padres de familia para mejorar la } \\
\text { infraestructura y servicios de los estudiantes. }\end{array}$} & $\begin{array}{l}\text { Totalmente en } \\
\text { desacuerdo }\end{array}$ & $5.3 \%$ \\
\hline & & De desacuerdo & $15.8 \%$ \\
\hline & & Indiferente & $21.1 \%$ \\
\hline & & De acuerdo & $36.8 \%$ \\
\hline & & Totalmente de acuerdo & $21.1 \%$ \\
\hline & & Total & $100 \%$ \\
\hline \multirow[t]{5}{*}{ BÁSICO } & \multirow{5}{*}{$\begin{array}{l}\text { 4. Aporta y gestiona recursos materiales } \\
\text { solicitados en la institución educativa, } \\
\text { así como también responde a la } \\
\text { invitación de docentes en razón de las } \\
\text { actividades que realiza su Hijo (a). }\end{array}$} & En desacuerdo & $15.8 \%$ \\
\hline & & Indiferente & $5.3 \%$ \\
\hline & & De acuerdo & $42.1 \%$ \\
\hline & & Totalmente de acuerdo & $36.8 \%$ \\
\hline & & Total & $100 \%$ \\
\hline
\end{tabular}

En cuanto al primer nivel evaluado como óptimo, un $57.9 \%$ de las personas encuestadas está de acuerdo con este nivel de participación, mientras que un $26.3 \%$ está totalmente de acuerdo. Con respecto al segundo nivel considerado como alto, 47.4\% está totalmente de acuerdo, mientras que un 42.1\% considera estar de acuerdo. Al comparar los porcentajes de este nivel con el resto, se puede decir que ambos porcentajes son significativos en cuanto a la participación de los padres, dado que implica el apoyo en la realización de tareas de sus hijos, así como elaboración de materiales didácticos necesarios en la institución educativa. Por otra parte, el tercer nivel de participación calificado como medio, es desglosado en los distintos grados de la escala likert, siendo

Incidencia de la participación ciudadana en educación en el contexto

20. salvadoreño 
así que: $36.8 \%$ está de acuerdo, $21.1 \%$ está totalmente de acuerdo, $21.1 \%$ es indiferente, $15.8 \%$ está en desacuerdo, y $5.3 \%$ está totalmente en desacuerdo. Finalmente el último nivel considerado como básico, señala que el $42.1 \%$ de las personas entrevistadas, considera aportar y gestionar recursos solicitados en la institución educativa y responder a la invitación de docentes; mientras que $36.8 \%$ considera estar de acuerdo, $15.8 \%$ en desacuerdo, y $5.3 \%$ es indiferente a este nivel. De acuerdo con esta información, podrá decirse que los padres de familia consideran tener una mayor participación de nivel óptimo y alto en la educación de sus hijos.

Por otra parte, la participación ciudadana en educación demanda la existencia de redes de comunicación entre los padres de familia e instituciones educativas, por lo que fue consultada la existencia de redes de comunicación entre la institución educativa y los padres de familia. De acuerdo a los datos, el 84.2\% cree que existen redes de comunicación, mientras que un $15.8 \%$ considera todo lo contrario. Entre las razones que aducían los padres de familia en cuanto a la oportuna comunicación entre ambos actores (padres de familia e instituciones educativas), señalaban la existencia de información de las actividades relacionadas con los estudiantes, redes de comunicación como asambleas de padres, reuniones con maestros, utilización de redes sociales, boletines educativos, circulares, entre otros. Por otra parte, quienes consideraban que no existía participación, manifestaban la falta de liderazgo de la institución educativa para mantener contacto eficiente con los padres de familia.

Tabla 8. Redes de comunicación entre padres y madres y la institución educativa.

\begin{tabular}{cc}
\hline \multirow{2}{*}{$\begin{array}{c}\text { ¿Considera que existen redes de comunicación en- } \\
\text { tre la institución educativa y los padres y(o) madres } \\
\text { de familia en la institución de su hijo(a)? }\end{array}$} \\
\hline $\mathrm{Si}$ & $84.2 \%$ \\
$\mathrm{No}$ & $15.8 \%$ \\
\hline Otros & $100.0 \%$ \\
\hline
\end{tabular}

Finalmente, en cuanto a la valoración de la incidencia de la participación ciudadana en educación, entendida como, la organización entre la comunidad, escuela, alumnos y demás, que ayuda a la planificación, seguimiento y valoración de las acciones educativas, en miras a mejorar la calidad educativa de los estudiantes, los padres de familia determinaron que esta era importante y necesaria. Los resultados se expresan en la siguiente tabla.

Tabla 9. La participación ciudadana y su incidencia en la actividad educativa.

\begin{tabular}{lcc}
\hline ¿Usted cree que la participación ciudadana en & \multicolumn{2}{c}{ Sí } \\
\cline { 2 - 3 } educación, entendida como, la organización entre la & Frecuencia & Porcentaje \\
\cline { 2 - 3 } comunidad, escuela, alumnos y demás, que ayuda & 19 & $100.0 \%$ \\
a la planificación, seguimiento y valoración de las & & \\
acciones educativas, en miras a mejorar la calidad & & \\
educativa de los estudiantes? & & \\
\hline
\end{tabular}




\section{Conclusión}

La información presentada anteriormente, tanto a nivel teórico como estadístico, permite entrever que el tema de participación es una herramienta de importancia que implica la colaboración entre actores, que buscan dar solución a determinado problema o situación. En cuanto a educación se refiere, el involucramiento de actores como escuela, padres de familia, alumnos, gobierno, entre otros, puede incidir en los procesos de índole educativa de los estudiantes, dado que convergen diferentes puntos de vista y aportes que ayudan a brindar una solución más completa del problema a resolver.

De acuerdo con los resultados de la encuesta realizada, el tema de participación ciudadana no es un tema ajeno para los padres y madres de familia ya a que existe una excelente valoración sobre el tema como método de promoción de la calidad educativa de los estudiantes, que involucra la organización entre la comunidad, escuela, alumnos y demás, para ayudar a la planificación, seguimiento y valoración de las acciones educativas. Este tipo de participación es relevante para la educación de sus hijos y, por tanto, se involucran, cooperan en las diferentes actividades relacionadas a su formación académica.

Los datos indican que este tipo de participación es realizada en mayor parte por el género femenino, por lo que deben buscarse mecanismos de inserción que promuevan una mayor diversidad en la participación, y así tanto padres como madres puedan verse involucrados en la educación de sus hijos. En base a la presente investigación, es notorio que existen aún desafíos que deben irse solventando con prontitud, siendo importante que exista comunicación efectiva entre los padres de familia e instituciones educativas, de tal forma que se evite que el proceso educativo se convierta en una especie de negocio, y que no provea de bienestar a la comunidad en general.

Si bien la investigación se concentró en un primer momento en la valoración de los padres de familia sobre el tema, no está demás también incentivar la participación de los estudiantes, dado que la educación es un tema que también compete a ellos y por tanto merecen brindar su opinión al respecto. Asimismo, los docentes, como las organizaciones de la sociedad civil, entre otros, deben ser incluidos en este tipo de procesos, dado que existe la posibilidad de retroalimentación entre sectores en vistas a mejorar las actividades escolares de los estudiantes.

En materia de política pública es importante una mayor acción por parte de las autoridades correspondientes ya que, si bien los esfuerzos se hacen notar, es necesario todavía la promoción de mecanismos de participación y comunicación entre escuela, padres de familia, alumnos. Al involucrar a los verdaderos actores, existe la posibilidad de establecer una educación integral y

Incidencia de la participación ciudadana en educación en el contexto

22. salvadoreño completa para todos, sin ningún tipo de distinción.

Naturalmente, la educación por sí sola no puede ayudar a resolver los problemas sociales existentes, pero si es una buena herramienta de inicio por donde los 
cambios pueden comenzar a darse. Cuando se accede a una educación de calidad existen mayores oportunidades de trabajo, mejores ingresos, acceso a servicios básicos, entre otros, que pueden traducirse eventualmente en una mejor calidad de vida de la población, por lo que es importante considerar este tipo de propuestas relacionadas a la participación ciudadana.

\section{Referencias}

CERCA (2004). Estudio cualitativo sobre la participación ciudadana en el mejoramiento de la calidad de la educación en cinco países latinoamericanos. [En línea] Disponible en: http://www.rinace.net/bliblio/Cerca\%202004.pdf

Córdova, R.; Macías, R.; Cruz, J. y Zechmeister, E. (2014). Cultura política de la democracia en El Salvador y en las Américas, 2014: Gobernabilidad democrática a través de 10 años del Barómetro de las Américas. San Salvador: FUNDAUNGO

Corporación de Desarrollo Social de Providencia (2016). Mecanismos de participación ciudadana en educación. Disponible en http://transparencia.cdsprovidencia. $\mathrm{cl} /$ mecanismos-de-participacion-ciudadana/mecanismos-de-participacionciudadana-en-educacion.

Corte de Cuentas de la República (2016). Corte de Cuentas y Ministerio de Educación Ianzan plan piloto de Contraloría Escolar. [En línea] Disponible en: http://www. cortedecuentas. gob.sv/?art $=1352$ \&title $=$ CORTE $\% 20$ DE $\% 20$ CUENTAS $\% 20 Y \% 20$ MINISTERIO\%20DE\%20EDUCACI\%D3N\%2OLANZAN\%20\%2OPLAN\%2OPILOTO\%20 DE\%20CONTRALOR\%CDA\%2OESCOLAR\&lang=es

Dueñas, L. y García, E. (2012). El estudio de la cultura de participación, aproximación a la demarcación del concepto. [En línea] Disponible en: http://www.razonypalabra. org.mx/N/N80/M80/07_DuenasGarcia_M80.pdf

FUNDE (2008). Mecanismos de participación ciudadana y transparencia en la gestión municipal: Guía para la implementación. [En línea] Disponible en: http://www. repo. funde.org/21/1/TR-001.pdf

Guillen, A.; Sáenz, K.; Badii, M. H. y Castillo, J. (2009), Origen, espacio y niveles de participación ciudadana. [En línea] Disponible en: http://www.spentamexico.org/ v4-n1/4(1)\%20179-193.pdf

Ministerio de Educación (2016). Dirección de Transparencia. Solicitud de Información número 0460 y 0461. San Salvador.

Secretaría de Educación Pública de México (2010). Qué es la participación social. [En línea] Disponible en: http://www.consejosescolares.sep.gob.mx/es/conapase/ Que_es_la_Participacion_Social_

Secretaría para Asuntos Estratégicos de la Presidencia (2014). Agenda Pro Participación Ciudadana en la Gestión Pública 2013-2014; Informe de Participación de Participación Ciudadana en la Gestión Pública 2013. San Salvador, El Salvador.

Tamayo, M. (2013). Participación ciudadana, políticas públicas y educación En América Latina y Ecuador. [En línea] Disponible en: http://www.oei.es/noticias /spip. php?article13155

Torres, Rosa María (2001) Participación ciudadana y educación. Una mirada amplia y 20experiencias en América Latina. [En línea] Disponible en: http://observatorio. sdhjgd.gob.hn/biblioteca-virtual/publicaciones/derechos-humanos/121participaci\%C3\%B3n-ciudadana-y-educaci\%C3\%B3n-una-mirada-amplia-y-20- 
experiencias-en-am\%C3\%A9rica-latina/file

Varela, L. (2014). La educación: importancia y pertinencia de la participación ciudadana. [En línea] Disponible en: http://observatorio.inie.ucr.ac.cr/attachments/article /28/ importancia\%20y\%20permanencia.pdf

Villareal, M. T. (2010). Participación ciudadana y políticas públicas. [En línea] Disponible en: http://www.cee-nl.org.mx/educacion/certamen_ensayo/decimo/MariaTeresa VillarrealMartinez.pdf 